Acta Cryst. (2002). A58 (Supplement), C41

\section{STRUCTURAL AND MECHANICAL CHARACTERISATION OF TiC/VC MULTILAYERS USING XRD, POLARIZED EXAFS AND NANOINDENTATION}

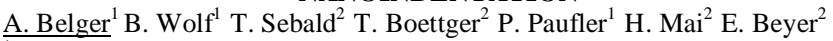

${ }^{1}$ Institut f. Kristallographie u. Festkoerperphysik der TU, D-01062 Dresden

${ }^{2}$ Fraunhofer-Institut f. Werkstoff- u. Strahlforschung, D-01277 Dresden

The superlattice hardening effect for tic/vc multilayers on the level of nanoindentations in relation to their structural parameters was investigated. Various multilayers of different period thicknesses were grown on sapphire (012) using pulsed laser deposition and targets of tic and vc. X-ray diffraction revealed the nanocrystalline structure of the thin layers. Moreover, a preferred orientation of the (110) planes of tic and vc parallel to the film surface was observed. Polarized fluorescence exafs experiments were carried out at hasylab. Considering the different weights of the in-plane and out-of-plane contributions from the nearest neighbors to the exafs signal the lattice parameters in-plane and out-of-plane of tic and vc were derived. These parameters exhibit a legible dependency on the period thickness of the tic/vcmultilayers, smaller values in-plane than out-of-plane and allow the determination of internal stresses in the multilayers. Nanoindentation experiments were carried out using a depth sensing electrostatic transducer (hysitron triboscope ${ }^{\circledR}$ ) and a Berkovich Indenter. Nanohardness and elastic modulus of the film/ substrate combination showed only a slight dependence on the contact indentation depth according to the behavior of a hard film (33 gpa - 39 gpa) on a hard substrate (30 gpa). The hardness of the tic/vc multilayers increased after high-temperature heat treatment $\left(5 \mathrm{~h}\right.$ at $\left.500^{\circ} \mathrm{c}\right)$ and decreased for the single-layer films. For the annealed films a maximum of the nanohardness of 39 gpa could be observed for a period thickness of about 5 $\mathrm{nm}$. Thus superlattice hardening on a nanoscale has been found in this system.

Keywords: MULTILAYERS EXAFS NANOHARDNESS
Acta Cryst. (2002). A58 (Supplement), C41

ELECTRON CRYSTALLOGRAPHY OF A ZEOLITE

T. Ohsuna ${ }^{1}$ Z. Liu ${ }^{2}$ O. Terasaki ${ }^{3}$ K. Hiraga ${ }^{1}$

${ }^{1}$ Institute For Materials Research, Tohoku University 2-1-1 Katahira, Aoba-Ku SENDAI 980-8577 JAPAN ${ }^{2}$ IMRAM, Tohoku Universiy ${ }^{3}$ Department of Physics, Tohoku Universiy

Transmission electron microscopy (TEM) is the most powerful technique to study nano-scale structural features of inorganic materials especially from a small crystallite with a sub-micron grain size. Some unknown crystal structures have been solved by the TEM. We have been developed electron crystallography to determine crystal structures of zeolites and meso-porous materials. In order to obtain structural solutions, relatively high voltage acceleration $(300 \mathrm{kV}$ or higher) and quantitative recording media (SSCCD or IP) are required. Framework structure of SSZ-48 (SFE) was solved by a combination of the direct method and electron diffraction intensity measurement obtained from selected area electron diffraction (SAED) patterns. This success indicates that, for applying electron intensity to the direct method, dynamical scattering of electrons in zeolite crystal can be neglected when specimen thickness is less than ca. $50 \mathrm{~nm}$. Namely, one can say that information of correlation between atom pairs in the framework is hold in observed diffraction intensities. Recently, a framework of BEC was retrieved by using high-resolution electron microscope (HREM) images and seven SAED patterns. The framework in a blurred potential distribution reconstructed from the HREM images were enhanced by using atom pair correlation obtained from a Patterson map, which was derived from the SAED patterns. Above two methods give only a feature of framework topology, but DLS-type calculation is helpful to refine atomic positions in the framework.

Keywords: ELECTRON CRYSTALLOGRAPHY ZEORITE TEM
Acta Cryst. (2002). A58 (Supplement), C41

\section{THE STRUCTURAL DISORDER IN THE ZSM-48 FAMILY OF} ZEOLITE MATERIALS

$\underline{\text { R. Lobo }}^{1}$ H. van Koningsveld ${ }^{2}$ P.J. Kooyman ${ }^{3}$

${ }^{1}$ University of Delaware Department of Chemical Engineering Colburn Lab. 150 Academy St. NEWARK DELAWARE 19716 USA ${ }^{2}$ Laboratory of Applied Chemistry and Catalysis, Delft University of Technology ${ }^{3}$ National Centre for HREM, Delft University of Technology

A detailed investigation of the disorder in zeolite ZSM-48 will be presented. Using powder X-ray diffraction patterns (XRD), simulations of faulted structures using DIFFaX, and Transmission Electron Microscopy (TEM) it is shown that ZSM-48 is not one but a family of materials with different degrees of disorder. The periodic building unit is a 10-ring pore formed from a rolledup 6-ring net. These pores can be arranged in two types layers: one in which consecutive pores are related by translations perpendicular to the pore direction, and a second in which the pores are related by translation perpendicular to the pore direction and translation of $1 / 2$ the unit cell dimensions along $b(4.2 \AA)$. These layers can be stacked following four distinct modes of stacking. Simulation of XRDs and TEM images of framework models built using these two layers and these stacking modes are in good agreement with experimental data. The structure of zeolite ZSM-48 is related to the structure of zeolites SSZ-31 and UTD-1 and relationships between the disorder in all these materials will be discussed.

\section{Keywords: ZSM-48 DISORDER ZEOLITE}

Acta Cryst. (2002). A58 (Supplement), C41

Ti SITES IN TS-1: RECENT DATA AND EVIDENCE

G. Artioli ${ }^{1,2}$ C. Lamberti ${ }^{3,4}$ G.L. Marra ${ }^{5}$

${ }^{1}$ Dipartimento di Scienze della Terra Università di Milano Via Botticelli 23 I20133 Milano Italy ${ }^{2}$ Centro CNR per lo Studio della Dinamica dei Sistemi Ambientali Via Mangiagalli 34 I-20133 Milano Italy ${ }^{3}$ Dipartimento di Chimica IFM Università di Torino Via P. Giuria 7 I-10125 Torino Italy ${ }^{4}$ INFM Unità di Torino Università ${ }^{5}$ Polimeri Europa S.p.A. Centro Ricerche Novara Istituto G. Donegani Via G. Fauser 4 I-28100 Novara

Recently, some works have reported direct experimental evidence of a nonrandom partitioning of $\mathrm{Ti}$ among the 12 independent $\mathrm{T}$ sites of the MFI framework of TS-1 by powder neutron diffraction. The neutron scattering contrast between $\mathrm{Ti}$ and $\mathrm{Si}$ has been exploited to locate the Ti atoms, which are just a small fraction of the framework sites, and to discriminate the framework substitutions from the Si site vacancies. As the maximum Ti content in TS- 1 is about $3.0 \mathrm{wt} \%$ the solution of the problem requires outstanding data and careful structure refinement strategies. Given that TS-1 is one of the most efficient heterogeneous catalysts in oxidation reactions, the recent experiments are welcome as they attempt to shed light on the relationship between TS-1 structural crystal-chemistry and chemical activity. The problems involved concern the mechanisms of insertion of $\mathrm{Ti}$ atoms in the defective silicalite framework, and their role in the catalytic processes.

The published results are apparently contradictory, as they identify different preferred sites of Ti. However each experimental procedure relies on different assumptions, such as the consistency in the synthesis procedures between different samples, or the assessment of the role of the $\mathrm{Si}$ vacancies on the $\mathrm{Ti}$ content obtained by the structure refinements. It may be argued that the discrepancies between the $\mathrm{T}$ sites identified to host Ti may be ascribed to a synthesis dependent insertion of Ti into the MFI framework. This hypothesis may also account for the discrepancy in the location of the Si vacancies in Tifree silicalite.

Keywords: SILICALITE, CATALYSIS, FRAMEWORK SUBSTITUTION 